\title{
Changes in Body Mass Index Following HAART Initiation among HIV- Infected Women in the Women's Interagency HIV Study
}

\section{Anjali Sharma $^{1 *}$, Shalanda A Bynum ${ }^{2}$, Michael F Schneider ${ }^{3}$, Christopher Cox ${ }^{3}$, Phyllis C Tien ${ }^{4}$, Ronald C Hershow ${ }^{5}$, Deborah Gustafson ${ }^{6}$} and Michael W Plankey

${ }^{1}$ Departments of Medicine, Albert Einstein College of Medicine, Bronx, NY, USA

${ }^{2}$ Social and Behavioral Sciences, Uniformed Services University of the Health Sciences, Bethesda, USA

${ }^{3}$ Epidemiology, Johns Hopkins Bloomberg School of Public Health, Baltimore, MD, USA

${ }^{4}$ Medicine, University of California at San Francisco, USA

${ }^{5}$ Epidemiology, University of Illinois at Chicago, USA

${ }^{6}$ Neurology, SUNY Downstate Medical Center, Brooklyn, NY, USA

${ }^{7}$ Medicine, Division of Infectious Diseases, Georgetown University Medical Center, Washington, DC, USA

\begin{abstract}
Objective: Examine changes in, and factors associated with changing body mass index (BMI) in women following highly active antiretroviral therapy (HAART) initiation.

Methods: 1177 HIV-infected Women's Interagency HIV Study participants who contributed 10,754 years of followup following HAART initiation were studied. Changes in median BMI up to 15 years following HAART initiation, and the highest and lowest BMI reached following HAART initiation were summarized by pre-HAART BMI category $(<18.5$ [underweight], 18.5- $<25.0$ [normal weight], $25.0-<30.0$ [overweight], 30.0-<40.0 [obese], and $\geq 40.0$ [morbidly obese]). Multivariate mixed effects ordinal logistic regression estimated the degree of association of each exposure of interest with post-HAART BMI.

Results: Before HAART, 39\% percent of women had normal BMI, $31 \%$ were overweight, $23 \%$ were obese, and $5 \%$ were morbidly obese. Following HAART initiation, median BMI change (per 5 years) was $0.21 \mathrm{~kg} / \mathrm{m}^{2}(90 \%$ confidence interval [Cl]: -1.33, 0.42) for those with normal pre-HAART BMI, $0.39 \mathrm{~kg} / \mathrm{m}^{2}(90 \% \mathrm{Cl}: 0.15,0.66)$ for overweight, $0.31 \mathrm{~kg} /$ $\mathrm{m}^{2}(90 \% \mathrm{Cl}:-1.18,0.67)$ for obese, and $-0.36 \mathrm{~kg} / \mathrm{m}^{2}$ for morbidly obese women. After initiating HAART, $40 \%$ with normal pre-HAART BMI became overweight at some point; of those overweight, $46 \%$ remained overweight and $47 \%$ became obese; $71 \%$ of obese women remained obese and $27 \%$ became morbidly obese. Each year of nucleoside analog reverse transcriptase inhibitor use was associated with a $3 \%$ decreased odds of reaching a higher BMI category (OR $0.97,95 \% \mathrm{Cl}: 0.95,0.99$ ), while each year of protease inhibitor or non-nucleoside analog reverse transcriptase inhibitor use were associated with a $6 \%(\mathrm{OR} 1.06,95 \% \mathrm{Cl}: 1.04,1.08)$ and $5 \%(\mathrm{OR} 1.05,95 \% \mathrm{Cl}: 1.01,1.08)$ increased odds of having a higher BMI category, respectively.
\end{abstract}

Conclusions: Although overweight and obesity are highly prevalent in this large cohort of HIV-infected, minority women, HAART use was associated with only a modest increase in BMI over time.

Keywords: Obesity; Body mass index; HIV; Women; HAART; Women's interagency HIV study

\section{Introduction}

In the general population, overweight and obesity have been associated with hypertension, dyslipidemia, cardiovascular disease, and insulin resistance-conditions that are particularly common among HIVinfected persons, and which are expected to become more prevalent as these individuals age $[1,2]$. Current prevalence estimates of overweight and obesity in HIV-infected women range from 58-78\%, and are similar to those seen among HIV-uninfected women [3-6]. Among HIVinfected populations, cross-sectional studies have shown associations of higher body mass index (BMI) with older age, African American race/ethnicity, and higher CD4 cell counts, whereas injection drug use, smoking, alcohol use, and depression have been associated with lower BMI [3,7-10]. A previous study summarizing cross-sectional data from the Women's Interagency HIV Study (WIHS) cohort found that markers of advanced HIV infection, including AIDS diagnosis, elevated HIV RNA levels, and low CD4 count, as well as history of antiretroviral therapy use were associated with lower BMI [3]. To expand the focus from cross-sectional studies to those that attempt to understand the relationship of antiretroviral therapy use and BMI over time particularly among populations disproportionately impacted by both HIV infection and obesity is needed. Published studies on the relationship between highly active antiretroviral therapy (HAART) use and weight gain have not yielded consistent results, and most have included only short-term HAART use, or have been conducted in military cohorts composed primarily of men [11-16]. Therefore, the purpose of this current study was to (1) examine the change in BMI from pre-HAART up to 15 years following HAART initiation by pre-HAART BMI; (2) describe the highest and lowest BMI attained following HAART initiation by preHAART BMI; and (3) determine the exposures related to a higher BMI following the initiation of HAART.

\section{Methods}

\section{Study population}

The WIHS is an ongoing, multicenter, observational study of women

*Corresponding author: Anjali Sharma, Albert Einstein College of Medicine, 1300 Morris Park Ave, Block BIdg \#305, Bronx, NY 10461, USA, Tel: 718-430-2067; Fax: 718-430-4035; E-mail: anjali.sharma@einstein.yu.edu

Received May 21, 2014; Accepted June 25, 2014; Published July 07, 2014

Citation: Sharma A, Bynum SA, Schneider MF, Cox C, Tien PC, et al. (2014) Changes in Body Mass Index Following HAART Initiation among HIV-Infected Women in the Women's Interagency HIV Study. J AIDS Clin Res 5: 323. doi:10.4172/21556113.1000323

Copyright: (C) 2014 Sharma A, et al. This is an open-access article distributed unde the terms of the Creative Commons Attribution License, which permits unrestricted use, distribution, and reproduction in any medium, provided the original author and source are credited. 
who are either HIV infected or at risk for HIV acquisition, established to carry out comprehensive investigations of the impact of HIV infection in women, including the clinical, laboratory and psychosocial aspects of HIV infection and the effectiveness and consequences of HAART.

A total of 3,766 including (2,791 HIV-infected women were enrolled in 1994-95 $(n=2,054)$ or 2001-02 $(n=737)$ from six sites (Bronx/Manhattan, Brooklyn, Chicago, Los Angeles, San Francisco and Washington DC). WIHS methods and baseline cohort characteristics have been described previously [17]. At each semi-annual visit, participants complete a physical examination and provide biological specimens and information on demographics, disease characteristics, and ARV use. The protocol was approved by the institutional review boards at each site, and all participants provided written informed consent.

Sixteen hundred and eleven HIV-infected women initiated HAART while enrolled in WIHS, of whom 1343 had at least two BMI measurements at visits in the five years prior to HAART initiation, a BMI measurement at the visit HAART was first reported and a BMI measurement for at least one visit following HAART initiation. The final study population was comprised of the 1177 women who had data on all exposures of interest (described below) at HAART initiation and at least one visit after HAART initiation. The 166 participants that were excluded because of missing exposure data had median pre-HAART BMI that was $1.1 \mathrm{~kg} / \mathrm{m}^{2}$ lower than the final study population.

\section{Body mass index}

BMI was determined at each visit by dividing measured body weight $(\mathrm{kg})$ by measured height ${ }^{2}\left(\mathrm{~m}^{2}\right)$ and categorized as: underweight $(<18.5)$, normal weight $(18.5$ to $<25.0)$, overweight $(25.0$ to $<30.0)$, obese $(30.0$ to $<40.0)$ and morbidly obese $(\geq 40.0)$ [18]. Average preHAART BMI was defined as the mean of the BMI measurements in the five years prior to HAART initiation; categorization of pre-HAART BMI into one of the five categories defined above was made based on this average value.

\section{Exposures}

In addition to examining the relationship between pre-HAART BMI and BMI following HAART initiation, we also examined the effects of enrollment period (1994-1995 vs. 2001-2002), age (per 5 years) at HAART initiation, race/ethnicity (Hispanic, Caucasian/Other vs. African-American), and level of education (high school graduate, some college, college graduate vs. non-high school graduate) at study entry. We included alcohol use ( $\geq 3$ vs. $<3$ drinks per week), cigarette smoking (former, current vs. never smoke), quality of life summary score (per 10 units calculated using the scores from six domains, i.e. physical functioning, role functioning, energy/fatigue, social functioning, pain and emotional well-being) based on an established algorithm using a shortened version of the Medical Outcome Study (MOS)-HIV [19,20], depressive symptomatology using the Center for Epidemiologic Studies Depression Scale (CESD $\geq 16$ vs. $<16)$ [21], CD4 cell count $(200$ to $<500$ cells $/ \mathrm{mm}^{3}, 100$ to $<200$ cells $/ \mathrm{mm}^{3},<100$ cells $/ \mathrm{mm}^{3}$ vs. $>500$ cells $/ \mathrm{mm}^{3}$ ), $\log _{10}$ HIV RNA, and history of a prior AIDS-defining illness (ADI) as time-varying predictors, incorporating the value at the visit prior to ascertainment of BMI. Use of marijuana cocaine, crack, heroin, or IDU since the last visit (yes to any vs. no to all) was time-varying, using the current value at the visit. Finally, to adjust for discontinuation of all ARV following HAART initiation, we used a time-varying indicator variable for whether or not ARV was reported since the last visit (no ARV vs. ARV) at each visit following HAART initiation.
At each visit, drug-years of ARV exposure were determined separately for nucleoside reverse transcriptase inhibitors (NRTI), protease inhibitors (PI), and non-nucleoside reverse transcriptase inhibitors (NNRTI). In addition, within the NRTI class, we determined the drug years of exposure separately for zidovudine, stavudine, abacavir, and tenofovir. At each visit the amount of exposure time (years) to each class of ARV was defined as the product of the number of drugs reported within a given class since the last visit and the time between the previous and current visits. We adjusted the time of exposure for self-reported adherence $(100 \%, 95 \%$ to $<100 \%, 75 \%$ to $<95 \%,>0 \%$ to $<75 \%$, and $0 \%$ ) by weighting the time of exposure by the midpoint $(100 \%, 97.5 \%, 85 \%, 37.5 \%, 0 \%)$ of the reported category. In all analyses, we used cumulative adherence-adjusted exposure to each class of ARV, by simply adding the adherence-adjusted exposure from the current visit to the total adherence-adjusted exposure from all previous visits. Drugs within a class were considered exchangeable and additive. Adherence data were collected beginning in October 1998; participants contributing visits to analyses prior to October 1998 were considered to be $100 \%$ adherent at these visits since $100 \%$ adherence was the most frequently reported adherence category following October 1998. At the baseline visit, we assumed that each drug reported was used for 0.5 years prior to the visit.

\section{Statistical analyses}

The median and inter-quartile range of BMI was used to summarize the distribution of BMI at each visit following HAART initiation separately for those with normal weight, those who were overweight, those who were obese, and those who were morbidly obese based on their pre-HAART initiation BMI category. A box-percentile plot, which shows more extreme percentiles of the distribution in addition to the standard median and quartiles, was used to summarize the distribution of average pre-HAART BMI. Quantile regression was used to model the median BMI and estimate the change in median BMI (per five years) following HAART initiation [22]. We estimated standard errors for $90 \%$ confidence intervals (CI) using bootstrap resampling with 500 samples [23]; samples were selected at the individual level, first randomly selecting individuals and then including all visits for each individual selected for a given sample.

To explore BMI change within each individual, we summarized the highest and lowest BMI category attained following HAART initiation by pre-HAART BMI category.

To evaluate the dynamics of BMI after HAART initiation, we determined the total number of person-years following HAART spent within each of the five BMI categories for each participant. Specifically, at each post-HAART initiation visit, we simply added $1 / 2$ of the difference in time between the current and previous visit to $1 / 2$ of the difference in time between the current and subsequent visit to estimate the time spent within a certain BMI category for a given visit. Person-years were then aggregated over all visits for each individual and then again over all individuals for each of the five BMI categories.

Multivariate mixed effects ordinal logistic regression models were used to estimate the association each exposure had with post-HAART BMI category. Odds ratios are the measure of association and are subject-specific since the model takes into account the subject-tosubject variation and is interpreted as the odds of a higher BMI category for a given exposure divided by the odds of a higher BMI category among those in the reference category. Ninety-five percent confidence intervals were used as a measure of precision. A two-sided p-value $<0.05$ was considered statistically significant. Statistical analyses were 
Citation: Sharma A, Bynum SA, Schneider MF, Cox C, Tien PC, et al. (2014) Changes in Body Mass Index Following HAART Initiation among HIVInfected Women in the Women's Interagency HIV Study. J AIDS Clin Res 5: 323. doi:10.4172/2155-6113.1000323

\begin{tabular}{|c|c|}
\hline Characteristic & Median (inter-quartile range) or $\%$ \\
\hline Date of HAART initiation & $\begin{array}{l}\text { February } 1998 \text { (March 1997, } \\
\text { February 2000) }\end{array}$ \\
\hline Cumulative NRTI use-years & $3.3(1.6,5.1)$ \\
\hline Cumulative zidovudine use-years & $1.0(0.5,1.9)$ \\
\hline Cumulative stavudine use-years & $0.5(0.0,1.0)$ \\
\hline Cumulative abacavir use-years & $0.0(0.0,0.0)$ \\
\hline Cumulative tenofovir use-years & $0.0(0.0,0.0)$ \\
\hline Cumulative PI use-years & $0.5(0.2,0.6)$ \\
\hline Cumulative NNRTI use-years & $0.0(0.0,0.4)$ \\
\hline Enrollment Period 1994-1995 & $91 \%$ \\
\hline Age, years ${ }^{a}$ & $38.7(33.6,44.1)$ \\
\hline $\begin{array}{l}\text { Race/Ethnicity } \\
\text { African-American } \\
\text { Hispanic } \\
\text { White } \\
\text { Other }\end{array}$ & $\begin{array}{r}56 \% \\
25 \% \\
16 \% \\
2 \% \\
\end{array}$ \\
\hline Alcohol Use $\geq 3$ drinks/week ${ }^{a}$ & $18 \%$ \\
\hline $\begin{array}{l}\text { Cigarette Smoking } \\
\text { Current } \\
\text { Former } \\
\text { Never } \\
\end{array}$ & $\begin{array}{l}51 \% \\
22 \% \\
27 \%\end{array}$ \\
\hline Marijuana usesince last visit & $18 \%$ \\
\hline $\begin{array}{l}\text { Injection drug, heroin, cocaine, or crack } \\
\text { use since last visit }\end{array}$ & $13 \%$ \\
\hline Quality of Life & $65.5(48.4,79.5)$ \\
\hline$C E S D \geq 16^{a}$ & $49 \%$ \\
\hline $\begin{array}{l}\text { Highest education level attained } \\
\text { Graduated college or higher } \\
\text { Some College } \\
\text { Completed High School } \\
\text { Did not complete high school }\end{array}$ & $\begin{array}{l}8 \% \\
25 \% \\
31 \% \\
37 \%\end{array}$ \\
\hline Have AIDS ${ }^{a}$ & $40 \%$ \\
\hline $\begin{array}{l}\text { CD4 cell count, cells } / \mathrm{mm}^{3 a} \\
\quad<100 \\
100-<200 \\
200-<500 \\
\geq 500\end{array}$ & $\begin{array}{l}15 \% \\
18 \% \\
51 \% \\
15 \%\end{array}$ \\
\hline $\log _{1}$ HIVRNA, copies/mla & $4.2(3.3,4.8)$ \\
\hline
\end{tabular}

aascertained from visit immediately prior to initiating HAART

Table 1: Participant characteristics at initial HAART visit, $\mathrm{N}=1177$. conducted using SAS 9.2 software (SAS Institute, Cary, NC).

\section{Results}

The study population of 1177 women contributed a total of 18,698 post-HAART initiation visits during 10,754 person-years of follow-up (median9.5 person-years [IQR 4.9-13.8]). Table 1 shows participant characteristics at the visit when HAART was first reported. The median date of HAART initiation was February 1998, with participants reporting a median of 3.3 years of cumulative NRTI use and six months of cumulative PI use. At the time of first visit with reported HAART, the median cumulative use reported for specific NRTIs was 1.0 years for zidovudine, 0.5 years for stavudine, 0.0 years for abacavir and 0.0 years for tenofovir. The women were a median 38.7 years of age, and reported high rates of current smoking (51\%) as well as depressive symptoms (49\%). Approximately one-third of women had a CD4 count below 200 $\mathrm{c} / \mathrm{mm}^{3}$ and $40 \%$ reported a history of ADI prior to initiating HAART.

The distribution of average pre-HAART initiation BMI was positively skewed with the largest $50 \%$ of values spread over a much wider interval than the smallest $50 \%$ (Figure 1). Median pre-HAART BMI was $26.3 \mathrm{~kg} / \mathrm{m}^{2}$. Thirty-nine percent had normal pre-HAART BMI, $31 \%$ were overweight, $23 \%$ were obese, and $5 \%$ were morbidly obese.

Figure 2 shows the median and inter-quartile range of BMI at each visit following HAART initiation, stratified by category of preHAART BMI. We did not include the 17 women who were underweight based on their pre-HAART BMI. In each pre-HAART BMI category, median BMI remained stable over time, following HAART initiation. Specifically, for women with pre-HAART BMI $18.5-<25.0$, the fiveyear change in median BMI following HAART initiation was $0.21 \mathrm{~kg} /$ $\mathrm{m}^{2}$ (90\% CI: $\left.-1.33,0.42\right)$, for overweight women $0.39 \mathrm{~kg} / \mathrm{m}^{2}(90 \%$ CI: $0.15,0.66)$, for obese women $0.31 \mathrm{~kg} / \mathrm{m}_{2}(90 \% \mathrm{CI}:-1.18,0.67)$, and morbidly obese women $0.36 \mathrm{~kg} / \mathrm{m}_{2},(90 \%$ CI: $-2.04,1.08)$.

As shown at the top of Table 2, the majority of women had a maximum BMI following HAART initiation that was either consistent with their pre-HAART BMI category or was one category higher. Specifically, of the 456 women with normal BMI prior to HAART

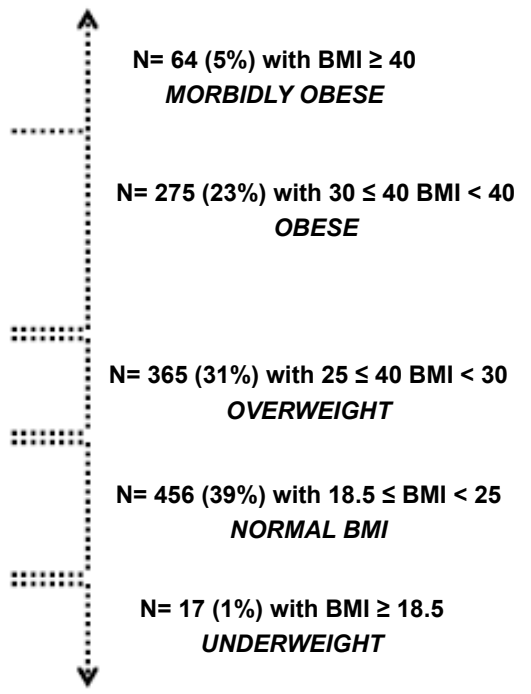

Figure 1: Box-percentile plot of mean pre-highly active antiretroviral therapy (HAART) initiation body mass index (BMI) values taken up to five years prior to HAART initiation. The central $95 \%$ of the distribution of values are shown. The minimum BMI was $15.2 \mathrm{~kg} / \mathrm{m}^{2}$ and the maximum BMI was $70.6 \mathrm{~kg} / \mathrm{m}^{2}$. 
Citation: Sharma A, Bynum SA, Schneider MF, Cox C, Tien PC, et al. (2014) Changes in Body Mass Index Following HAART Initiation among HIVInfected Women in the Women's Interagency HIV Study. J AIDS Clin Res 5: 323. doi:10.4172/2155-6113.1000323

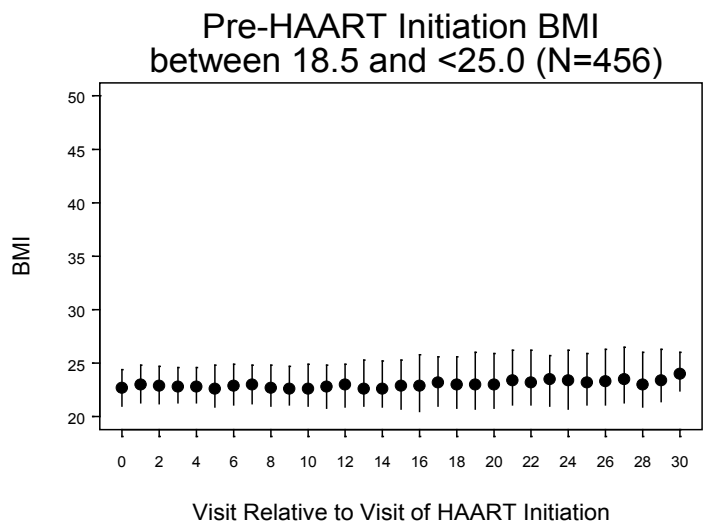

Pre-HAART Initiation BMI

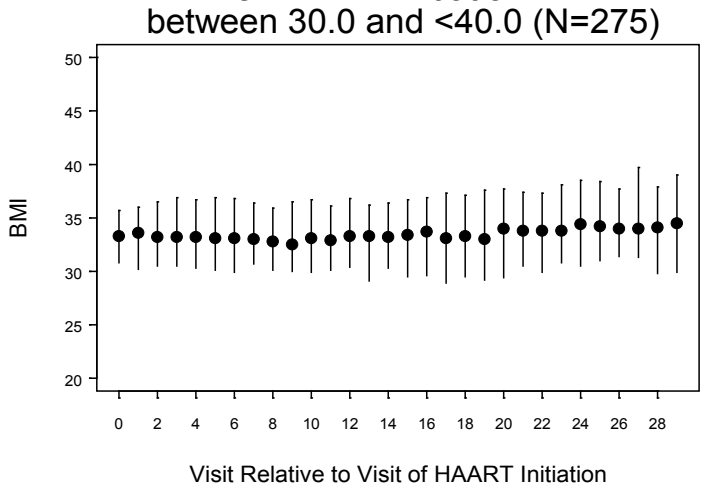

Pre-HAART Initiation BMI between 25.0 and $<30.0(\mathrm{~N}=365)$

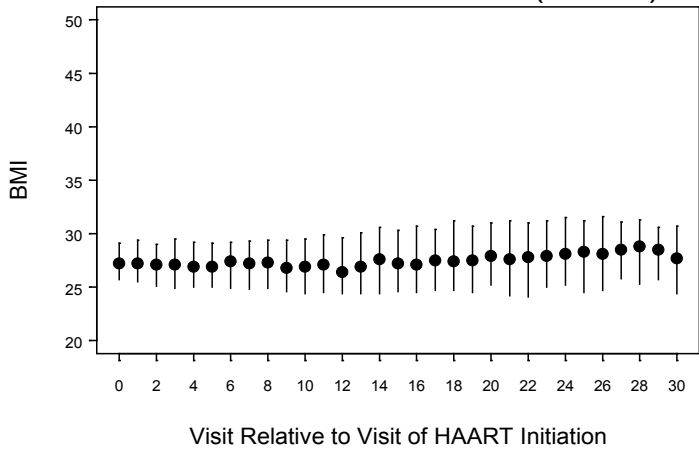

Pre-HAART Initiation BMI

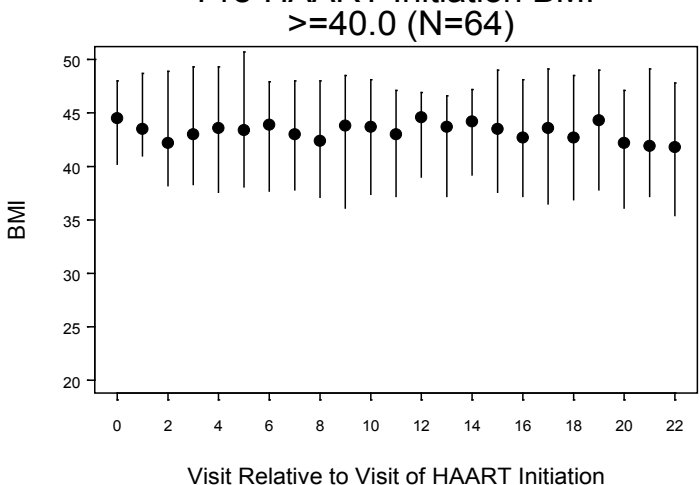

Figure 2: Median and inter-quartile range of body mass index (BMI) at each visit after HAART initiation stratified by pre-HAART initiation BMI category.

\begin{tabular}{|c|c|c|c|c|c|c|}
\hline \multirow[b]{2}{*}{ Pre-HAART mean BMI $\left(\mathrm{kg} / \mathrm{m}^{2}\right)$} & \multirow[b]{2}{*}{ No. } & \multicolumn{5}{|c|}{ Highest post-HAART BMI $\left(\mathrm{kg} / \mathrm{m}^{2}\right)$ reached } \\
\hline & & Underweight $(<18.5)$ & Normal $(18.5-<25.0)$ & Overweight $(25.0-<30.0)$ & Obese $(30.0-<40.0)$ & Morbidly Obese $(\geq 40.0)$ \\
\hline Underweight $(<18.5)$ & 17 & $\begin{array}{c}4 \\
(24 \%)\end{array}$ & $\begin{array}{c}10 \\
(59 \%)\end{array}$ & $\begin{array}{c}3 \\
(18 \%)\end{array}$ & $\begin{array}{c}0 \\
(0 \%)\end{array}$ & $\begin{array}{c}0 \\
(0 \%)\end{array}$ \\
\hline Normal $(18.5-<25.0)$ & 456 & $\begin{array}{c}3 \\
(1 \%)\end{array}$ & $\begin{array}{c}215 \\
(47 \%)\end{array}$ & $\begin{array}{c}183 \\
(40 \%)\end{array}$ & $\begin{array}{c}53 \\
(12 \%)\end{array}$ & $\begin{array}{c}2 \\
(<1 \%)\end{array}$ \\
\hline Overweight $(25.0-<30.0)$ & 365 & $\begin{array}{c}1 \\
(<1 \%)\end{array}$ & $\begin{array}{c}12 \\
(3 \%)\end{array}$ & $\begin{array}{c}168 \\
(46 \%)\end{array}$ & $\begin{array}{c}172 \\
(47 \%)\end{array}$ & $\begin{array}{c}12 \\
(3 \%)\end{array}$ \\
\hline Obese $(30.0-<40.0)$ & 275 & $\begin{array}{c}0 \\
(0 \%)\end{array}$ & $\begin{array}{c}0 \\
(0 \%)\end{array}$ & $\begin{array}{c}8 \\
(3 \%)\end{array}$ & $\begin{array}{c}194 \\
(71 \%)\end{array}$ & $\begin{array}{c}73 \\
(27 \%)\end{array}$ \\
\hline Morbidly Obese $(\geq 40.0)$ & 64 & $\begin{array}{c}0 \\
(0 \%)\end{array}$ & $\begin{array}{c}0 \\
(0 \%)\end{array}$ & $\begin{array}{c}0 \\
(0 \%)\end{array}$ & $\begin{array}{c}4 \\
(6 \%)\end{array}$ & $\begin{array}{c}60 \\
(94 \%)\end{array}$ \\
\hline \multirow[t]{2}{*}{$\underline{\text { Total }}$} & 1177 & $\begin{array}{c}8 \\
(1 \%)\end{array}$ & $\begin{array}{c}237 \\
(20 \%)\end{array}$ & $\begin{array}{c}362 \\
(31 \%)\end{array}$ & $\begin{array}{c}423 \\
(36 \%)\end{array}$ & $\begin{array}{c}147 \\
(12 \%)\end{array}$ \\
\hline & & \multicolumn{5}{|c|}{ Lowest post-HAART BMI $\left(\mathrm{kg} / \mathrm{m}^{2}\right)$ reached } \\
\hline Pre-HAART mean BMI $\left(\mathrm{kg} / \mathrm{m}^{2}\right)$ & No. & Underweight $(<18.5)$ & Normal $(18.5-<25.0)$ & Overweight $(25.0-<30.0)$ & Obese $(30.0-<40.0)$ & $\begin{array}{c}\text { Morbidly Obese ( } \geq \\
40.0)\end{array}$ \\
\hline Underweight $(<18.5)$ & 17 & $\begin{array}{c}16 \\
(94 \%)\end{array}$ & $\begin{array}{c}1 \\
(6 \%)\end{array}$ & $\begin{array}{c}0 \\
(0 \%)\end{array}$ & $\begin{array}{c}0 \\
(0 \%)\end{array}$ & $\begin{array}{c}0 \\
(0 \%)\end{array}$ \\
\hline Normal $(18.5-<25.0)$ & 456 & $\begin{array}{c}107 \\
(23 \%)\end{array}$ & $\begin{array}{c}333 \\
(73 \%)\end{array}$ & $\begin{array}{c}15 \\
(3 \%)\end{array}$ & $\begin{array}{c}1 \\
(<1 \%)\end{array}$ & $\begin{array}{c}0 \\
(0 \%)\end{array}$ \\
\hline Overweight $(25.0-<30.0)$ & 365 & $\begin{array}{c}24 \\
(7 \%)\end{array}$ & $\begin{array}{c}197 \\
(54 \%)\end{array}$ & $\begin{array}{c}133 \\
(36 \%)\end{array}$ & $\begin{array}{c}11 \\
(3 \%)\end{array}$ & $\begin{array}{c}0 \\
(0 \%)\end{array}$ \\
\hline Obese $(30.0-<40.0)$ & 275 & $\begin{array}{c}4 \\
(1 \%)\end{array}$ & $\begin{array}{c}59 \\
(21 \%)\end{array}$ & $\begin{array}{c}101 \\
(37 \%)\end{array}$ & $\begin{array}{c}108 \\
(39 \%)\end{array}$ & $\begin{array}{c}3 \\
(1 \%)\end{array}$ \\
\hline Morbidly Obese $(\geq 40.0)$ & 64 & $\begin{array}{c}0 \\
(0 \%)\end{array}$ & $\begin{array}{c}4 \\
(6 \%)\end{array}$ & $\begin{array}{c}9 \\
(14 \%)\end{array}$ & $\begin{array}{c}27 \\
(42 \%)\end{array}$ & $\begin{array}{c}24 \\
(38 \%)\end{array}$ \\
\hline$\underline{\text { Total }}$ & 1177 & $\begin{array}{c}151 \\
(13 \%)\end{array}$ & $\begin{array}{c}594 \\
(50 \%)\end{array}$ & $\begin{array}{c}258 \\
(22 \%)\end{array}$ & $\begin{array}{c}147 \\
(12 \%)\end{array}$ & $\begin{array}{c}27 \\
(2 \%)\end{array}$ \\
\hline
\end{tabular}

Table 2: Highest and Lowest body mass index (BMI) category reached following highly active antiretroviral therapy (HAART) initiation stratified by pre-HAART mean BM category. Percentages given are row percentages. 
Citation: Sharma A, Bynum SA, Schneider MF, Cox C, Tien PC, et al. (2014) Changes in Body Mass Index Following HAART Initiation among HIVInfected Women in the Women's Interagency HIV Study. J AIDS Clin Res 5: 323. doi:10.4172/2155-6113.1000323

initiation, $47 \%$ had their highest BMI following HAART initiation in the normal category and $40 \%$ became overweight for at least one visit: among 365women who were overweight prior to HAART initiation, similar proportions remained overweight $(46 \%)$ or became obese (47\%); of the 275 obese women initiating HAART, $71 \%$ remained at most obese and $27 \%$ gained enough weight after HAART initiation to put them in the morbidly obese category. In total, $79 \%$ of women were observed at least once following HAART initiation with BMI $\geq 25.0 \mathrm{~kg} /$ $\mathrm{m}^{2}$. The bottom of Table 2 shows the lowest post-HAART BMI category attained following HAART initiation. Fifty-four percent of women who were overweight at HAART initiation attained normal BMI at some point, whereas only $21 \%$ of obese women and $6 \%$ of morbidly obese women attained a normal BMI. Overall, $50 \%$ of women were observed at least once following HAART initiation with BMI between 18.5 and
$<25.0$, although this group was largely comprised (333 of 594) of those who had normal BMI prior to initiating HAART.

Table 3 shows the total person-years spent in each BMI category following HAART initiation stratified by pre-HAART BMI category. The majority of time post-HAART initiation was spent in the same BMI category as the pre-HAART value (highlighted diagonal in Table 3 ), although the movement between BMI categories was not trivial. For example, overweight women spent $50 \%$ of their person-years in the same overweight BMI category, and roughly equivalent amounts of time in the normal weight (25\%) and obese (23\%) categories following HAART initiation. Unfortunately, among those who were morbidly obese prior to HAART initiation, nearly all of the time following HAART was spent obese $(30 \%)$ or morbidly obese $(66 \%)$. Similarly, those who were underweight prior to HAART initiation spent a majority $(62 \%)$ of their

\begin{tabular}{|c|c|c|c|c|c|c|c|}
\hline \multirow[b]{2}{*}{ Pre-HAART mean BMI $\left(\mathrm{kg} / \mathrm{m}^{2}\right)$} & \multirow[b]{2}{*}{ No. } & \multirow[b]{2}{*}{$\begin{array}{l}\text { Total No. Person-Years } \\
\text { Following HAART Initiation }\end{array}$} & \multicolumn{5}{|c|}{ Total No. Person - Years in each BMI category $\left(\mathrm{kg} / \mathrm{m}^{2}\right)$} \\
\hline & & & $\begin{array}{l}\text { Underweight } \\
\quad(<18.5)\end{array}$ & $\begin{array}{c}\text { Normal } \\
(18.5-<25.0)\end{array}$ & $\begin{array}{l}\text { Overweight } \\
(25.0-<30.0)\end{array}$ & $\begin{array}{c}\text { Obese } \\
(30.0-<40.0)\end{array}$ & $\begin{array}{l}\text { Morbidly Obese } \\
\quad(\geq 40.0)\end{array}$ \\
\hline Underweight $(<18.5)$ & 17 & 124.6 & $76.8(62 \%)$ & $45.9(37 \%)$ & $1.9(2 \%)$ & $0.0(0 \%)$ & $0.0(0 \%)$ \\
\hline Normal(18.5 - <25.0) & 456 & 4011.4 & $212.7(5 \%)$ & $2759.4(69 \%)$ & $887.7(22 \%)$ & $150.4(4 \%)$ & $1.2(0 \%)$ \\
\hline Overweight $(25.0-<30.0)$ & 365 & 3449.8 & $33.6(1 \%)$ & $861.7(25 \%)$ & $1732.2(50 \%)$ & $793.9(23 \%)$ & $28.4(1 \%)$ \\
\hline Obese $(30.0-<40.0)$ & 275 & 2587.5 & $2.3(0 \%)$ & $97.6(4 \%)$ & $486.3(19 \%)$ & $1700.7(66 \%)$ & $300.6(12 \%)$ \\
\hline Morbidly Obese $(\geq 40.0$ ) & 64 & 580.5 & $0.0(0 \%)$ & $3.0(1 \%)$ & $20.2(4 \%)$ & $174.9(30 \%)$ & $382.4(66 \%)$ \\
\hline
\end{tabular}

Table 3: Total Person-Time in each category of body mass index (BMI) following highly active antiretroviral therapy (HAART) initiation stratified by pre-HAART mean BMI category.

\begin{tabular}{|c|c|c|}
\hline Characteristic & Adjusted Odds Ratio & 95\% Confidence Interval \\
\hline $\begin{array}{l}\text { Pre-HAART BMI category } x \text { Years foll } \\
\text { Underweight }(<18.5) \\
\text { Normal }(18.5 \text { to }<25.0) \\
\text { Overweight }(25.0 \text { to }<30.0) \\
\text { Obese }(30.0 \text { to }<40.0) \\
\text { Morbidly Obese }(\geq 40.0)\end{array}$ & $\begin{array}{l}0.18 \\
1.19 \\
1.05 \\
1.10 \\
0.92 \\
\end{array}$ & $\begin{array}{l}0.09,0.37 \\
0.98,1.44 \\
0.87,1.26 \\
0.91,1.33 \\
0.67,1.25\end{array}$ \\
\hline $\begin{array}{l}\text { ARV discontinued at visit } \\
\text { Yes } \\
\text { No }\end{array}$ & $\begin{array}{l}0.66 \\
\text { Ref }\end{array}$ & $\begin{array}{c}0.57,0.76 \\
\text { Ref }\end{array}$ \\
\hline Cumulative NRTI-years, per year & 0.97 & $0.95,0.99$ \\
\hline Cumulative PI-years, per year & 1.06 & $1.04,1.08$ \\
\hline Cumulative NNRTI-years, per year & 1.05 & $1.01,1.08$ \\
\hline $\begin{array}{l}\text { Enrollment Period } \\
\text { 1994-1995 } \\
2001-2002\end{array}$ & $\begin{array}{l}0.66 \\
\text { Ref }\end{array}$ & $\begin{array}{c}0.26,1.68 \\
\text { Ref }\end{array}$ \\
\hline Age at HAART initiationa , per 5 years & 0.89 & $0.75,1.05$ \\
\hline $\begin{array}{l}\text { Race/Ethnicity } \\
\text { Hispanic } \\
\text { White/Other } \\
\text { African-American }\end{array}$ & $\begin{array}{l}0.80 \\
0.20 \\
\text { Ref }\end{array}$ & $\begin{array}{l}0.41,1.54 \\
0.10,0.42 \\
\quad \text { Ref }\end{array}$ \\
\hline $\begin{array}{l}\text { Alcohol use } \\
\qquad 3 \text { drinks/week } \\
\quad<3 \text { drinks/week }\end{array}$ & $\begin{array}{l}0.87 \\
\text { Ref }\end{array}$ & $\begin{array}{c}0.75,1.00 \\
\text { Ref }\end{array}$ \\
\hline $\begin{array}{l}\text { Cigarette smoking } \\
\text { Current } \\
\text { Former } \\
\text { Never }\end{array}$ & $\begin{array}{l}0.58 \\
1.17 \\
\text { Ref }\end{array}$ & $\begin{array}{l}0.37,0.91 \\
0.75,1.84 \\
\quad \text { Ref }\end{array}$ \\
\hline $\begin{array}{l}\text { Marijuana use since last visit } \\
\text { Yes } \\
\text { No }\end{array}$ & $\begin{array}{l}0.57 \\
\text { Ref }\end{array}$ & $\begin{array}{c}0.49,0.67 \\
\text { Ref }\end{array}$ \\
\hline $\begin{array}{l}\text { Injection drug, heroin, cocaine, or crac } \\
\text { Yes } \\
\text { No }\end{array}$ & $\begin{array}{l}0.32 \\
\text { Ref }\end{array}$ & $\begin{array}{c}0.26,0.38 \\
\text { Ref }\end{array}$ \\
\hline Quality of life, per 10 units & 1.00 & $0.97,1.03$ \\
\hline $\begin{array}{l}\text { Depressive symptomatology } \\
\text { CESD } \geq 16 \\
\text { CESD }<16\end{array}$ & $\begin{array}{l}0.89 \\
\text { Ref }\end{array}$ & $\begin{array}{c}0.80,0.99 \\
\text { Ref }\end{array}$ \\
\hline
\end{tabular}




\begin{tabular}{|c|c|c|}
\hline $\begin{array}{l}\text { Highest education level attained } \\
\text { Graduated college or higher } \\
\text { Some college } \\
\text { Completed high school } \\
\text { Did not complete high school }\end{array}$ & $\begin{array}{l}0.31 \\
0.95 \\
1.53 \\
\text { Ref }\end{array}$ & $\begin{array}{l}0.10,0.93 \\
0.46,1.93 \\
0.79,2.96 \\
\text { Ref }\end{array}$ \\
\hline $\begin{array}{l}\text { History of AIDS } \\
\text { Yes } \\
\text { No }\end{array}$ & $\begin{array}{r}0.80 \\
\text { Ref }\end{array}$ & $\begin{array}{c}0.66,0.96 \\
\text { Ref }\end{array}$ \\
\hline $\begin{array}{l}\text { CD4 cell count }\left(\text { cells } / \mathrm{mm}^{3}\right) \\
<100 \\
100-<200 \\
200-<500 \\
\geq 500\end{array}$ & $\begin{array}{l}0.24 \\
0.53 \\
0.73 \\
\text { Ref }\end{array}$ & $\begin{array}{l}0.19,0.30 \\
0.45,0.63 \\
0.66,0.82 \\
\text { Ref }\end{array}$ \\
\hline HIV RNA copies/ml, per $\log _{10}$ & 0.93 & $0.89,0.97$ \\
\hline
\end{tabular}

HAART, highly active antiretroviral therapy; NRTI, nucleoside reverse transcriptase inhibitory; PI, protease inhibitor; NNRTI, non-nucleoside reverse transcriptase inhibitor

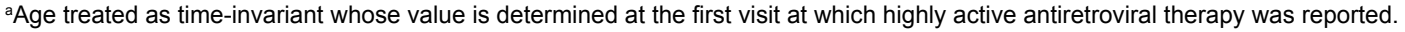

Table 4: Multivariate longitudinal ordinal logistic regression analysis of body mass index following initiation of highly active antiretroviral therapy among 1177 women contributing a total of 18,698 person-visits during a total of 10,754 years of follow-up. Odds ratios represent the odds of attaining a higher BMI category.

post-HAART initiation time underweight.

Table 4 shows the degree of association each exposure had with the odds of being in a higher BMI category following HAART initiation. With the exception of the lowest pre-HAART BMI category, there was no significant relationship over time between the pre-HAART BMI category and the odds of being in a higher BMI category following HAART. Discontinuing all ARV following HAART initiation was associated with a $34 \%$ decreased odds $(\mathrm{OR}=0.66,95 \% \mathrm{CI}$ : $0.57,0.76)$ of reaching a higher BMI category. And also remove all of the $=$ in the following sentence: Each year of zidovudine use was associated with a $14 \%$ reduced odds of reaching a higher BMI category $(\mathrm{OR}=0.86,95 \%$ CI: $0.83,0.89)$ and each year of stavudine use was associated with a $13 \%$ reduced odds of reaching a higher $\mathrm{BMI}$ category $(\mathrm{OR}=0.87,95 \% \mathrm{CI}$ : $0.84,0.91)$, while each year of abacavir and tenofovir use was associated with a $4 \%(\mathrm{OR}=1.04,95 \% \mathrm{CI}: 1.01,1.08)$ and $7 \%(\mathrm{OR}=1.07,95 \% \mathrm{CI}$ : $1.03,1.12$ ) increased odds of having a higher BMI category, respectively (data not shown). Each year of zidovudine use was associated with a $14 \%$ reduced odds of reaching a higher BMI category $(\mathrm{OR}=0.86,95 \%$ CI: $0.83,0.89$ ) and each year of stavudine use was associated with a $13 \%$ reduced odds of reaching a higher $\mathrm{BMI}$ category $(\mathrm{OR}=0.87,95 \% \mathrm{CI}$ : $0.84,0.91)$, while each year of abacavir and tenofovir use was associated with a $4 \%(\mathrm{OR}=1.04,95 \% \mathrm{CI}: 1.01,1.08)$ and $7 \%(\mathrm{OR}=1.07,95 \% \mathrm{CI}$ : $1.03,1.12)$ increased odds of having a higher BMI category, respectively (data not shown). Having lower HIV RNA levels, higher CD4 cell count, and absence of history of AIDS were all associated with greater odds of a higher BMI category. Being a white woman, current smoker, user of either marijuana or any hard drugs, having greater depressive symptomatology and being a college graduate were all associated with a lower likelihood of attaining a higher BMI category. Sensitivity analyses which included only person-visits with complete self-reported adherence data (without any estimation of adherence) showed similar results.

\section{Discussion}

The majority of HIV-infected women in this study were overweight or obese prior to the initiation of HAART, yet BMI remained relatively stable post-HAART initiation, with the majority of time post-HAART initiation in the same BMI category as pre-HAART. Most women did not progress past one BMI category higher than their initial preHAART BMI classification, although those in the highest weight categories in particular were unlikely to lose substantial amounts of weight. When examining specific ARV class, we found that while NRTI exposure was associated with reduced odds of greater BMI, both PI use and NNRTI use were associated with a greater likelihood of higher
BMI. In particular, use of thymidine analogs was associated with a reduced odds of higher BMI, which may be a result of direct effects of these agents on adipose tissue; these agents may be leading to regional fat loss, such as lipoatrophic changes occurring primarily in extremity fat, and less associated with overall weight loss; alternatively their effects on BMI may not be apparent when considering HAART use as a whole as they may be counterbalanced by the effects of PI or NNRTI use on BMI change. Moreover, women who discontinued all ARV at their most recent visit were less likely to experience an increase in BMI, suggesting that stopping HAART may have a short-term effect on BMI change. We have not analyzed data on reasons for stopping individual medications, so it is difficult to interpret this effect very precisely. Our findings suggest that HAART initiation has only modest long-term effects on BMI change.

Published studies on the relationship between weight and HAART use have reported either modest changes in weight with HAART use, or found no association between HAART use and weight. In a crosssectional study conducted in an urban population, no relationship was found between those who were ARV naïve, currently on HAART, or currently on PIs and BMI, although NRTI use was not specifically examined [4]. Likewise, a retrospective study of male members of the military did not find a relationship between current HAART use, duration of HAART or PI use, or current use of specific antiretrovirals and obesity [11]. Short-term studies of weight change and HAART initiation conducted in predominantly male subjects in the early HAART era have shown modest weight gain immediately following HAART initiation among antiretroviral naïve subjects, followed by either stabilization over time or a return to baseline levels [13-15]; however decreases or no changes in weight were seen in antiretroviralexperienced subjects [15]. Tate et al. reported rapid weight gain in the first six months of HAART among previously naïve Southern U.S. patients which was sustained at 24 months, with greater increases in BMI observed in those with regimens containing boosted PIs [16]. Our data are consistent with the findings of Justman et al., who found that in the WIHS cohort between 1999 and 2004, waist and hip circumference and BMI did not significantly change among HIV-infected women, $48 \%$ of whom were on HAART [24]. Most similar to our findings, in a longitudinal study of early diagnosed HIV conducted in a predominantly male, military cohort, $62 \%$ of patients gained weight after HIV diagnosis, however the amount of weight gain was modest, such that among those who gained weight, mean BMI increase was $2.3 \mathrm{~kg} / \mathrm{m}^{2}$ [12]. In that study, longer cumulative NRTI exposure (most notably thymidine analogs as well as didanosine and zalcitabine) were associated with smaller increase in weight gain; NNRTIs and PIs were 
Citation: Sharma A, Bynum SA, Schneider MF, Cox C, Tien PC, et al. (2014) Changes in Body Mass Index Following HAART Initiation among HIVInfected Women in the Women's Interagency HIV Study. J AIDS Clin Res 5: 323. doi:10.4172/2155-6113.1000323

associated with greater increases in weight gain per year of exposure. When restricting analyses to those diagnosed with HIV in the HAART era and initiating HAART, NRTIs had a trend towards less weight gain; neither NNRTIs nor PIs were associated with weight gain [12].

In our study, measures of reduced HIV disease severity such as higher CD4 count, lower HIV RNA levels, and lack of an AIDS defining illness were associated with an increase dodds of being in a higher BMI category. These findings are consistent with other published data showing associations between higher BMI and greater CD4 cell counts and CD4 nadir, as well as reduced HIV disease progression in studies conducted early in the HIV epidemic [3,4,11,12,25-27]. Unsurprisingly, others have observed greater short-term increases in BMI among those with low CD4 counts [16,27]. While weight gain might be expected in the short-term period after HAART initiation as part of a return to health phenomenon, particularly in those with advanced disease, weight gain over extended time periods appears more likely to occur in those with less severe markers of HIV disease. Because our study included up to 15 years of prospective data on BMI, measures of HIV disease severity, and detailed information on use of antiretroviral therapy, we are able to more clearly understand the temporal relationships between weight gain and use of HAART, not only with initiation of HAART, but also with continuation of long-term, stable antiretroviral therapy. Our findings that women with well-controlled HIV are more likely to have a higher BMI category than those with advanced markers of HIV disease suggest that this weight gain may reflect factors unrelated to HIV disease, which might include poor diet, lack of physical activity, or genetic factors, although we were unable to measure those potential contributors in this study.

Wasting, which was commonly experienced by people living with HIV early in the epidemic is now very infrequent, and in our study less than $2 \%$ of women were underweight, whereas the majority were overweight or obese, including $5 \%$ who were morbidly obese $[11,28,29]$. As obesity has become a common experience in the general population, the identification, evaluation, and treatment of overweight and obesity should be incorporated as a routine part of primary care, regardless of HIV status. A recent systematic review and meta-analysis by Flegal et al. found that when compared with those of normal weight in the general U.S. population of men and women, obesity or morbid obesity (but not overweight) was associated with higher all-cause mortality [30]. Studies conducted among HIV-infected populations earlier in the HIV epidemic have suggested a protective effect of overweight on mortality, however it is unclear whether the effect extends into the current era of potent antiretroviral therapy [26,27,31]. Moreover, overweight and obesity are associated with hypertension, dyslipidemia, cardiovascular disease, and insulin resistance, conditions which are particularly common in HIV-infected persons, and in the general population weight loss is recommended for those with BMI $>25$ to lower blood pressure in those with hypertension, lower blood glucose in those with type 2 diabetes, and lower elevated levels of total cholesterol, LDL-cholesterol, and triglycerides, and raise low levels of HDL-cholesterol in those with dyslipidemia [32-34]. Targeted weight control efforts are warranted to reduce co-morbidities associated with both obesity and chronic HIV infection, particularly as these individuals advance in age.

Our study had several limitations. Because our cohort included only women with HIV infection, results may not be generalizable to HIV-infected men, as weight change and degrees of change on ART may differ between men and women. Because our focus was on overall BMI change associated with HAART use, we did not evaluate changes in body fat distribution associated with HAART. As with all observational studies, our findings are subject to possible unmeasured confounding. We were unable to measure the potential contributions of diet or lack of physical activity to weight gain because data were not collected on these characteristics over time in the WIHS cohort. Finally, the design of this cohort study, with study visits conducted every six months, only allows us to coarsely define exposure times to ARV classes and specific medications within a class.

In conclusion, long-term HAART use itself does not appear to significantly increase the risk of overweight and obesity in women with HIV-infection. However, reducing the impact of overweight and obesity should be encouraged among minority women with HIV in order to mitigate their risks for obesity-related health consequences as they age.

\section{Acknowledgements}

Data in this manuscript were collected by the Women's Interagency HIV Study (WIHS). The contents of this publication are solely the responsibility of the authors and do not represent the official views of the National Institutes of Health (NIH). WIHS (Principal Investigators): Bronx WIHS (Kathryn Anastos), U01-AI-035004 Brooklyn WIHS (Howard Minkoff and Deborah Gustafson), U01-AI-031834 Chicago WIHS (Mardge Cohen), U01-AI-034993; Metropolitan Washington WIHS (Mary Young), U01-Al-034994; Connie Wofsy Women's HIV Study, Northern California (Ruth Greenblatt, Bradley Aouizerat, and Phyllis Tien), U01-Al-034989 WIHS Data Management and Analysis Center (Stephen Gange and Elizabeth Golub), U01-AI-042590; Southern California WIHS (Alexandra Levine and Marek Nowicki), U01-HD-032632 (WIHS I - WIHS IV). The WIHS is funded primarily by the National Institute of Allergy and Infectious Diseases (NIAID), with additional co-funding from the Eunice Kennedy Shriver National Institute of Child Health and Human Development (NICHD), the National Cancer Institute ( $\mathrm{NCl}$ ), the National Institute on Drug Abuse (NIDA), and the National Institute on Mental Health (NIMH) Targeted supplemental funding for specific projects is also provided by the National Institute of Dental and Craniofacial Research (NIDCR), the National Institute on Alcohol Abuse and Alcoholism (NIAAA), the National Institute on Deafness and other Communication Disorders (NIDCD), and the NIH Office of Research on Women's Health. WIHS data collection is also supported by UL1-TR000004 (UCSF CTSA). Research reported in this publication was also supported by the National Institute of Arthritis and Musculoskeletal and Skin Diseases of the National Institutes of Health under Award Number 1K23AR061993-01A1to A.S.

\section{Financial support}

The WIHS is funded by the National Institute of Allergy and Infectious Diseases (U01-Al-35004, U01-Al-31834, U01-Al-34994, U01-Al-34989, U01-Al-34993, and U01-Al-42590) and by the Eunice Kennedy Shriver National Institute of Child Health and Human Development (U01-HD-32632). The study is co-funded by the National Cancer Institute, the National Institute on Drug Abuse, and the National Institute on Deafness and Other Communication Disorders. Funding is also provided by the National Center for Research Resources (UCSF-CTSI Grant Number UL1 RR024131).

\section{References}

1. Ekali LG, Johnstone LK, Echouffo-Tcheugui JB, Kouanfack C, Dehayem MY, et al. (2013) Fasting blood glucose and insulin sensitivity are unaffected by HAART duration in Cameroonians receiving first-line antiretroviral treatment Diabetes Metab 39: 71-77.

2. Buchacz K, Baker RK, Palella FJ Jr, Shaw L, Patel P, et al. (2013) Disparities in prevalence of key chronic diseases by gender and race/ethnicity among antiretroviral-treated HIV-infected adults in the US. Antivir Ther 18: 65-75.

3. Boodram B, Plankey MW, Cox C, Tien PC, Cohen MH, et al. (2009) Prevalence and correlates of elevated body mass index among HIV-positive and HIVnegative women in the Women's Interagency HIV Study. AIDS Patient Care STDS 23: 1009-1016.

4. Amorosa V, Synnestvedt M, Gross R, Friedman H, MacGregor RR, et al. (2005) A tale of 2 epidemics: the intersection between obesity and HIV infection in Philadelphia. J Acquir Immune Defic Syndr 39: 557-561.

5. Tedaldi EM, Brooks JT, Weidle PJ, Richardson JT, Baker RK, et al. (2006) Increased body mass index does not alter response to initial highly active antiretroviral therapy in HIV-1-infected patients. J Acquir Immune Defic Syndr 43: 35-41.

6. Centers for Disease Control and Prevention (2011) Behavioral Risk Factor Surveillance System Survey. Atlanta, GA: U.S. Department of Health and Human Services, Centers for Disease Control and Prevention. 
Citation: Sharma A, Bynum SA, Schneider MF, Cox C, Tien PC, et al. (2014) Changes in Body Mass Index Following HAART Initiation among HIVInfected Women in the Women's Interagency HIV Study. J AIDS Clin Res 5: 323. doi:10.4172/2155-6113.1000323

Page 8 of 8

7. Ferrando SJ, Rabkin JG, Lin SH, McElhiney M (2005) Increase in body cell mass and decrease in wasting are associated with increasing potency of antiretroviral therapy for HIV infection. AIDS Patient Care STDS 19: 216-223.

8. Karmon SL, Moore RD, Dobs AS, Keruly J, Barnett S, et al. (2005) Body shape and composition in HIV-infected women: an urban cohort. HIV Med 6: 245-252.

9. Silva M, Skolnik PR, Gorbach SL, Spiegelman D, Wilson IB, et al. (1998) The effect of protease inhibitors on weight and body composition in HIV-infected patients. AIDS 12: 1645-1651

10. Wand H, Ramjee G (2013) High prevalence of obesity among women who enrolled in HIV prevention trials in KwaZulu-Natal, South Africa: healthy diet and life style messages should be integrated into HIV prevention programs. BMC Public Health 13: 159

11. Crum-Cianflone N, Tejidor R, Medina S, Barahona I, Ganesan A (2008) Obesity among patients with HIV: the latest epidemic. AIDS Patient Care STDS 22: 925-930

12. Crum-Cianflone N, Roediger MP, Eberly L, Headd M, Marconi V, et al. (2010) Increasing rates of obesity among HIV-infected persons during the HIV epidemic. PLoS One 5: e10106.

13. Gallant JE, Staszewski S, Pozniak AL, DeJesus E, Suleiman JM, et al. (2004) Efficacy and safety of tenofovir DF vs stavudine in combination therapy in antiretroviral-naive patients: a 3-year randomized trial. JAMA 292: 191-201.

14. Mallon PW, Miller J, Cooper DA, Carr A (2003) Prospective evaluation of the effects of antiretroviral therapy on body composition in HIV-1-infected men starting therapy. AIDS 17: 971-979.

15. Shikuma CM, Zackin R, Sattler F, Mildvan D, Nyangweso P, et al. (2004) Changes in weight and lean body mass during highly active antiretroviral therapy. Clin Infect Dis 39: 1223-1230.

16. Tate T, Willig AL, Willig JH, Raper JL, Moneyham L, et al. (2012) HIV infection and obesity: where did all the wasting go? Antivir Ther 17: 1281-1289.

17. Bacon MC, von Wyl V, Alden C, Sharp G, Robison E, et al. (2005) The Women's Interagency HIV Study: an observational cohort brings clinical sciences to the bench. Clin Diagn Lab Immunol 12: 1013-1019.

18. National Institutes of Health (1998) Clinical Guidelines on the Identification, Evaluation, and Treatment of Overweight and Obesity in Adults--The Evidence Report. Obesity Research 6 : 51S-209S

19. Bozzette SA, Hays RD, Berry SH, Kanouse DE, Wu AW (1995) Derivation and properties of a brief health status assessment instrument for use in HIV disease. J Acquir Immune Defic Syndr Hum Retrovirol 8: 253-265.

20. Liu C, Weber K, Robison E, Hu Z, Jacobson LP, et al. (2006) Assessing the effect of HAART on change in quality of life among HIV-infected women. AIDS Res Ther 3: 6.

21. Radloff LS, Rae DS (1979) Susceptibility and precipitating factors in depression: sex differences and similarities. J Abnorm Psychol 88: 174-181.

22. Koenker R, Hallock K (2001) Quantile Regression. J Econ Perspect 15: 143 156 .

23. Cox C, Chu H, Schneider MF, Muñoz A (2007) Parametric survival analysis and taxonomy of hazard functions for the generalized gamma distribution. Stat Med 26: $4352-4374$

24. Justman JE, Hoover DR, Shi Q, Tan T, Anastos K, et al. (2008) Longitudina anthropometric patterns among HIV-infected and HIV-uninfected women. J Acquir Immune Defic Syndr 47: 312-319.

25. Palenicek JP, Graham NM, He YD, Hoover DA, Oishi JS, et al. (1995) Weight loss prior to clinical AIDS as a predictor of survival. Multicenter AIDS Cohort Study Investigators. J Acquir Immune Defic Syndr Hum Retrovirol 10: 366-373.

26. Jones CY, Hogan JW, Snyder B, Klein RS, Rompalo A, et al. (2003) Overweight and human immunodeficiency virus (HIV) progression in women: associations HIV disease progression and changes in body mass index in women in the HIV epidemiology research study cohort. Clin Infect Dis 37: S69-80.

27. Shor-Posner G, Campa A, Zhang G, Persaud N, Miguez-Burbano MJ, et al (2000) When obesity is desirable: a longitudinal study of the Miami HIV-1infected drug abusers (MIDAS) cohort. J Acquir Immune Defic Syndr 23: 81-88.

28. Lakey W, Yang LY, Yancy W, Chow SC, Hicks C (2013) Short communication: from wasting to obesity: initial antiretroviral therapy and weight gain in HIVinfected persons. AIDS Res Hum Retroviruses 29: 435-440.

29. Hodgson LM, Ghattas H, Pritchitt H, Schwenk A, Payne L, et al. (2001) Wasting and obesity in HIV outpatients. AIDS 15: 2341-2342.

30. Flegal KM, Kit BK, Orpana H, Graubard BI (2013) Association of all-cause mortality with overweight and obesity using standard body mass index categories: a systematic review and meta-analysis. JAMA 309: 71-82.

31. Shuter J, Chang CJ, Klein RS (2001) Prevalence and predictive value of overweight in an urban HIV care clinic. J Acquir Immune Defic Syndr 26: 291 297.

32. Bray GA (1985) Complications of obesity. Ann Intern Med 103: 1052-1062.

33. Wilson PW, D'Agostino RB, Sullivan L, Parise H, Kannel WB (2002) Overweight and obesity as determinants of cardiovascular risk: the Framingham experience. Arch Intern Med 162: 1867-1872.

34. NHLBI Obesity Education Initiative (2000) The Practical Guide: Identification, Evaluation, and Treatment of Overweight and Obesity in Adults. 